\title{
Development of Serotonin-Containing Neurons in Drosophila Mutants Unable to Synthesize Serotonin
}

\author{
Ana Maria Vallés and Kalpana White \\ Department of Biology, Brandeis University, Waltham, Massachusetts 02254
}

We have initiated a study of the CNS of mutant Drosophila melanogaster larvae carrying a genetic deletion of the gene $D d c$ that encodes the enzyme dopa decarboxylase (DDC). The two major objectives of this study were (1) to ascertain that the DDC encoded by the gene $D d c$ was the only decarboxylase utilized in serotonin (5HT)-containing neurons and (2) to determine the effect of DDC deficiency on the development of 5HT-immunoreactive neurons. CNSs of wild-type larvae and of larvae genetically deficient for the gene $D d c$ were processed for serotonin immunocytochemistry using a monoclonal antibody against 5HT. The pattern of 5HT immunoreactivity in the wild-type and the Ddc-deficient CNS is compared. In contrast to the wild-type, 5HT immunoreactivity is absent in the Ddc-deficient CNSs. The lack of immunocytochemically detectable 5HT in the mutant CNSs is consistent with the idea that the DDC encoded by the gene $D d c$ is utilized in 5HT-containing neurons.

To study the development of neurons committed to the 5HT differentiation pathway in the absence of 5HT, we used a second biochemical property characteristic of 5HT-containing neurons, the ability to take up 5HT. CNSs from mutant animals were incubated in exogenous $5 \mathrm{HT}$ and the accumulated $5 \mathrm{HT}$ detected immunocytochemically. Neurons capable of selective 5HT uptake were present in the mutant CNSs in the same pattern as the 5HT-immunoreactive neurons in the wild-type CNS. This result suggests that the presumed inability to synthesize $5 \mathrm{HT}$ does not preclude differentiation of other normal biochemical properties of 5HT-containing neurons.

Although it is clear that a variety of intrinsic and extrinsic signals acting in proper sequence is required for the development and maintenance of the precise architecturc and conncctivity of ncurons, the exact nature of these signals has remained elusive. The speculation that a classical neurotransmitter could act as a causal signal in neuronal morphogenesis and synaptogenesis is attractive because of the parsimonious integration it provides between ncural function and development (for a review, see Harris, 1981). Recently, Haydon et al. (1984) have demonstrated that the biogenic amine, serotonin $(5 \mathrm{HT})$, can inhibit neurite outgrowth of individual, identified, regenerating neurons in cell cultures of the snail Helisoma. A causal developmental role would be firmly established for 5HT if the tissue culture events observed by Haydon et al. (1984) are representative of the in vivo events during normal development.

\footnotetext{
Received July 25, 1985; revised Oct. 28, 1985; accepted Oct. 30, 1985

We are grateful to Dr. T. R. F. Wright for sharing with us the mutant strains and many tips on the biology of the mutant animals. We thank Dr. Eve Marder for stimulating discussions and critically reading the manuscript. We thank Michael Nusbaum, Dan Rosen, and Teresa Hurteau for their comments on the manuscript. We appreciate the suggestions from reviewers of the manuscript on the 5 HT permeability problem. This work was supported by NIH Grant GM31503. A.M.V. is a predoctoral trainee supported by the U.S. Department of Health and Human Services Training Grant NRSA GM-07122.

Correspondence should be addressed to $\mathrm{K}$. White at the above address.

Copyright (C) 1986 Society for Neuroscience $0270-6474 / 86 / 051482-10 \$ 02.00 / 0$
}

We have initiated a study of 5HT-containing and catecholamine-containing neurons in the fruit fly Drosophila melanogaster (Budnick et al., 1985; Vallés and White, 1985; White and Vallés, 1985). One of the incentives to study biogenic amine systems in Drosophila is provided by the potential in this organism to study CNSs that are genetically deficient in two important amines, 5HT and dopamine (Livingstone and Tempel, 1983; White and Vallés, 1985; Wright, 1977). Animals with a genetic deficiency in the biosynthesis of 5HT and dopamine can be generated by the use of mutations in the gene $D d c$, which codes for the enzyme dopa decarboxylase (DDC) (Wright et al., $1976 \mathrm{a}, \mathrm{b})$. In Drosophila, DDC is necessary in the synthetic pathway of the neurotransmitters 5HT and dopamine (Livingstone and Tempel, 1983). Our long-term goal is to determine the impact of the absence of 5HT and dopamine on the development and maintenance of specific neurons.

We have previously localized 5HT immunoreactivity in the wild-type larval CNS and shown that the larval 5HT-immunoreactive neurons are first discerned in the late embryo (Vallés and White, 1985; White and Vallés, 1985). In this paper we investigate the development of 5HT-containing neurons in the CNSs of larvae that carry genetic deletions of the $D d c$ gene and thus are lacking the DDC enzyme activity. Specifically, we address the following questions: (1) Is the mutant CNS devoid of any immunocytochemically detectable 5HT? (2) What is the fate of the mutant neurons that are normally committed to the 5 HT differentiation pathway?

\section{Materials and Methods}

\section{Fly cultures and strains}

All fly strains were raised at $25^{\circ} \mathrm{C}$ on standard cornmeal-molassesagar-yeast medium. The mutant chromosomes used are listed below. For more complete descriptions of mutations, see Lindsley and Grell (1968).

$D f(2 L)$ 130: A cytologically visible deletion, entirely removing $D d c$ (Wright et al., 1976a). It is marked with $r d o p r c n$.

$D d c^{n 27}$ : A 2 kilobase $(\mathrm{kb})$ deletion removing the two $5^{\prime}$ exons of $D d c$ and $0.5 \mathrm{~kb}$ of $5^{\prime}$ flanking DNA (J. Hirsh, personal communication). It is marked with $p r$.

CyO: A second chromosome balancer, marked with $\mathrm{dp}^{(n)} \mathrm{Cy} \mathrm{pr} \mathrm{cn}^{2}$.

\section{Generation of animals deficient for the gene Ddc}

Genetic cross. To generate animals that were deficient in DDC activity $\left(D f D d c\right.$ ), we used two $D d c$ deletions, $D f(2 L) 130$ and $D d c^{n 27}$. The genetic cross was $D f(2 L) / 30 / C y O \times D d c^{n 27} / C y O$. The genotypes of the progeny of this cross and their properties are listed below:

$D f(2 L) 130 / D d c^{n 27}: 25 \%$ of the progeny; $D d c$ deficient. Most $D f D d c$ mutant cmbryos normally die at the embryo larval boundary and do not hatch. The mutant pharate larvae can be readily recognized in late embryogenesis ( $20-22 \mathrm{hr}$ ) because of their incompletely sclerotized mouth hooks and setae. 

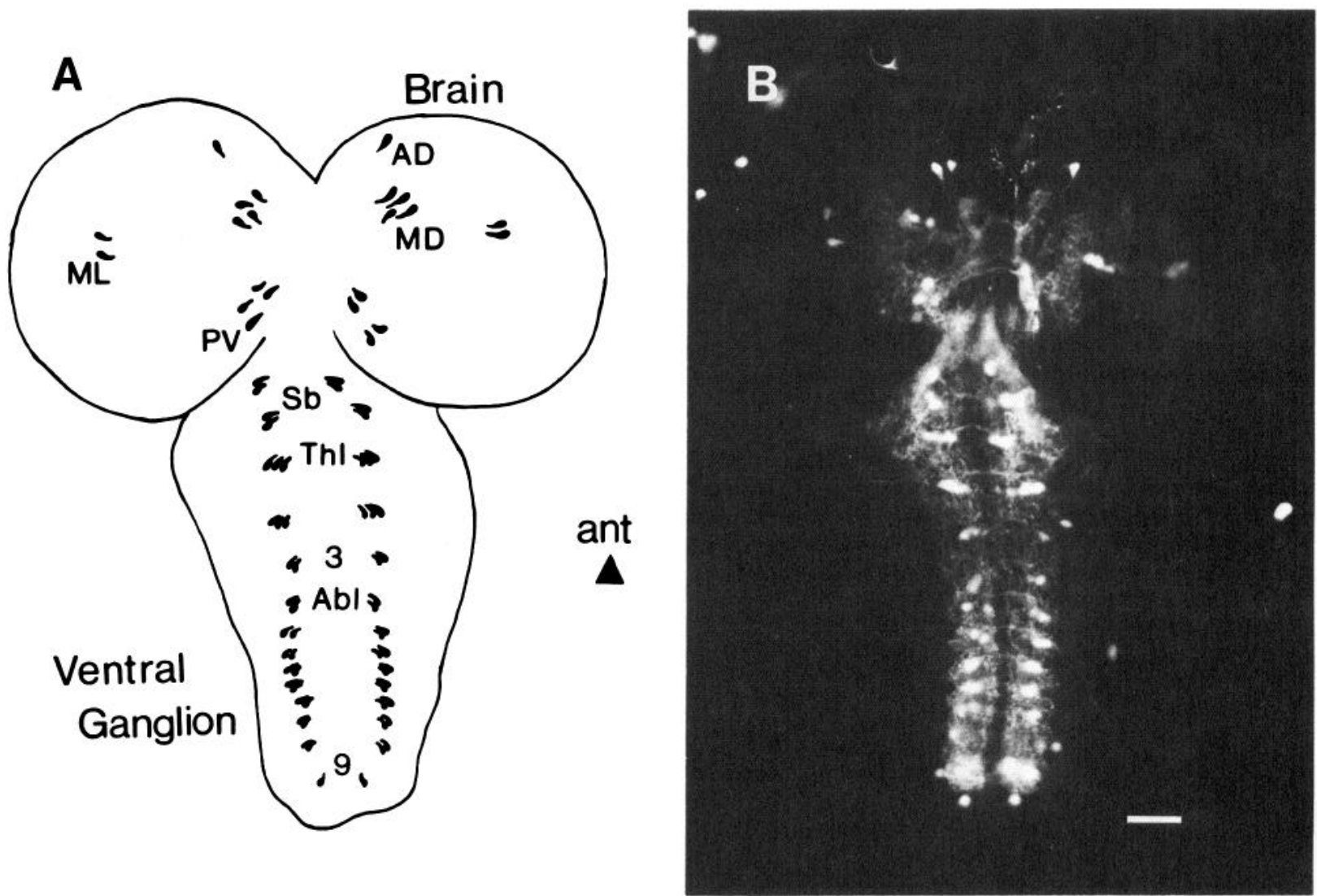

Figure 1. Serotonin-immunoreactive neurons in the third instar wild-type larval CNS. $A$, Schematic tracing adapted from whole-mount preparations of larval CNSs. For a description of the individual neuron clusters AD, MD, ML, PV, Th1-3, and Ab1-9, see Table 1. Immunoreactivity in the neuropil and fibers leaving the CNS are not drawn. $B$, Photomicrograph of the third instar wild-type larval CNS showing the 5HT-immunoreactive neurons visualized after immunofluorescent staining. Notice the regular pattern of 5HT-immunoreactive neurons in the ventral ganglion. ant, Anterior. Scale bar, $50 \mu \mathrm{m}$.

$D f(2 L) 130 / C y O$ and $D d c^{n 27} / C y O: 50 \%$ of the progeny; carry one wildtype $D d c$ gene. The heterozygotes carrying one wild-type $D d c$ gene develop normally. We have used them as control animals in all experiments.

$\mathrm{CyO} / \mathrm{CyO}: 25 \%$ of the progeny; homozygous $\mathrm{CyO}$. The $\mathrm{CyO}$ homo-

Table 1. Serotonin-immunoreactive neurons in the CNS of third instar larvae

\begin{tabular}{|c|c|c|}
\hline Group & $\begin{array}{l}\text { No. cell } \\
\text { bodies }\end{array}$ & Localization \\
\hline \multicolumn{3}{|c|}{ Brain lobes } \\
\hline $\mathrm{AD}$ & 1 & Anterior dorsal cortex, close to midline \\
\hline MD & $4-5$ & $\begin{array}{l}\text { Dorsal cortex, immediately below } \mathrm{AD} \\
\text { cluster, very close to midline }\end{array}$ \\
\hline ML & 2 & $\begin{array}{l}\text { Medial lateral cortex, adjacent to the } \\
\text { optic lobes }\end{array}$ \\
\hline PV & 4 & $\begin{array}{l}\text { Posterior near the ventral cortex } \\
\text { neuropil interface }\end{array}$ \\
\hline \multicolumn{3}{|c|}{ Ventral ganglion } \\
\hline $\mathrm{Sb}$ & $2-4$ & Subesophageal \\
\hline Th1 & 3 & Prothoracic \\
\hline Th2 & 3 & Mesothoracic \\
\hline Th3 & 2 & Metathoracic \\
\hline Abl-8 & 2 & First to eighth abdominal neuromere \\
\hline $\mathrm{Ab} 9$ & 1 & Ninth abdominal neuromere \\
\hline
\end{tabular}

zygotes are also embryonic lethal. However, they have darkly pigmented mouth hooks and thus are readily distinguished from the unhatched $D f D d c$ embryos.

\section{Egg collections}

To obtain mutant animals in sufficient numbers and to rear them under defined conditions, broods were allowed to lay eggs for a $2 \mathrm{hr}$ period on sucrose-agar ( $5 \%$ sucrose, $1.5 \%$ agar) plates topped with a drop of live yeast. The eggs were allowed to develop for $21-23 \mathrm{hr}$. By this time a majority of the control embryos ( $D d c$ heterozygotes) had already hatched. The unhatched embryos were first dechorionated by treatment with $50 \%$ Chlorox for 2-3 min and then were rinsed in $70 \%$ ethanol and distilled water.

Df Ddc larvae. The dechorionated embryos, exhibiting mutant phenotype, were mechanically liberated by teasing the vitellin membrane with a sharp tungsten needle. The mutant larvae were reared in small plastic vials $(40 \times 23.5 \mathrm{~mm})$ containing a special medium of the following composition: agar (Difco), 0.4\%; sucrose (Fisher), $5.0 \%$; yeast extract (Difco), 5.0\%; inactivated yeast (Red Star), 2.0\%. To retard microbial growth, $0.5 \mathrm{ml}$ of propionic acid-phosphoric acid (9:1) was added per $100 \mathrm{ml}$ of the medium.

The development of the mechanically hatched mutant larvae was monitored. The larval instars were determined on the basis of the morphology of the anterior spiracles (Bodenstein, 1950). Their survival index was defined as follows: survival index $=$ no. of larvae reaching third instar stage/no. mechanically hatched.

\section{Immunocytochemistry}

To visualize the $5 \mathrm{HT}$-containing neurons, the indirect immunofluorescence method was used. The larval CNSs were dissected in $\mathrm{Ca}^{2+}$ free ice-cold Drosophila Ringer's ( $\mathrm{NaCl}, 130 \mathrm{~mm} ; \mathrm{KCl}, 4.7 \mathrm{~mm} ; \mathrm{MgCl}_{2}, 1.8$ 
$\left.\mathrm{mm} ; \mathrm{KH}_{2} \mathrm{PO}_{4}, 0.74 \mathrm{~mm} ; \mathrm{Na}_{2} \mathrm{HPO}_{4}, 0.35 \mathrm{~mm}\right)$. The dissected tissues were fixed in $4 \%$ paraformaldehyde in $0.1 \mathrm{~m}$ phosphate buffer $(\mathrm{pH} 7.2)$. The details of the immunocytochemistry are described elsewhere (White et al., in press). Two primary 5HT antibodies were used. For the majority of the studies presented in this paper, an anti-5HT monoclonal supernatant (concentrated 40/50x) purchased from Accurate Chemical and Scientific Corporation (catalog no. MAS055, clone no. YC5/45) was used at dilution 1:200. For some of the initial studies, an anti-5HT serum purchased from Immunonuclear Corporation was used at dilution 1:250. The secondary antibody was goat anti-rat IgG or goat antirabbit IgG conjugated with fluorescein isothiocyanate purchased from Cappel Laboratories (dilution 1:20).

\section{HT uptake}

Larval CNSs were dissected in Drosophila Ringer's (NaCl, $130 \mathrm{~m} ; \mathrm{KCl}$, $4.7 \mathrm{~mm} ; \mathrm{CaCl}_{2}, 1.8 \mathrm{~mm} ; \mathrm{KH}_{2} \mathrm{PO}_{4}, 0.74 \mathrm{~mm} ; \mathrm{Na}_{2} \mathrm{HPO}_{4}, 0.35 \mathrm{~mm}$ ). Dissected CNSs were incubated at room temperature in Ringer's containing $10^{-6}$ м 5HT creatine sulfate (Sigma). At the end of $1 \mathrm{hr}$, tissues were rinsed $3 \times$ over a $15 \mathrm{~min}$ period in ice-cold $\mathrm{Ca}^{2+}$-free Ringer's. CNSs were then fixed and processed for immunocytochemistry.

Whole-mounts were examined under fluorescent optics, and for each $\mathrm{CNS}$, the presence or absence of individual neuronal clusters as revealed by fluorescent staining was recorded. The individual neuronal clusters are described in Table 1 .

In experiments to allow for better accessibility of the amine to the CNS, third instar larval CNSs were severed so as to frec onc brain lobe. The free brain lobes and the intact control CNSs were incubated in the presence of $5 \mathrm{HT}$ and processed for immunocytochemistry as described above.

\section{Results}

\section{HT-immunoreactive neurons in Drosophila larval CNS}

A specific pattern of $5 \mathrm{HT}$-containing neurons is revealed using anti-5HT serum in whole-mount preparations of the larval CNS (White and Vallés, 1985). Figure $1 A$ is a tracing of the third instar larval CNS onto which the somata of the 5HT-immunoreactive cells are drawn. The tracing is adapted from preparations similar to the one shown in the light photomicrograph of the fluorescently labeled larval CNS whole-mount (Fig. $1 B$ ). In Table 1 we provide the cell numbers and approximate localization within the cellular cortex for each neuron cluster.

In the paired larval brain hemispheres, about $225 \mathrm{HT}$-immunoreactive neurons are observed. These are arranged in four clusters. In the ventral ganglion, which in Drosophila is a compound ganglion consisting of subesophageal, thoracic, and abdominal neuromeres, every segment contains immunoreactive cell bodies (a total of about 58 neurons). The 5HT immunoreactivity is reproducible and bilaterally symmetrical (Fig. 1). In particular we would like to draw attention to the pattern of the 5HT-immunoreactive cells in the ventral ganglion, for which our current interpretation is described below. For these cells the regularity of the pattern allows direct comparison of different samples (Fig. 1).

The anteriorly located subesophageal region contains four 5HT-immunoreactive cells arranged in two clusters. The prothoracic and the mesothoracic hemisegments each contain a cluster composed of three neurons (triplet). The third thoracic hemisegment, the metathorax, contains a pair of cells (doublet). Just posterior to the metathoracic doublet, a row of eight doublets is usually present, followed by a single cell at the posterior end (Fig. 1). We interpret this pattern as a doublet per abdominal hemisegment, with the last hemisegment containing only one 5HT-immunoreactive cell.

In the ventral ganglion, the somata of the 5HT-containing neurons are located in the ventral cellular rind. As a rule these neurons send their projections to the contralateral side. Axons from each cell within a cluster seem to fasciculate. In immunoperoxidase-labeled sectioned material, the primary processes seem to travel along the cortex-neuropil interface to the con- tralateral side (A. M. Vallés, unpublished observations). The axons cross to the contralateral side and turn dorsally into the neuropil region, where their dendrites arborize (A. M. Vallés, unpublished observations).

This pattern of 5HT-containing neurons is already present in the newly hatched larvae and, at least in its more global aspects, is maintained throughout larval stages. This point is illustrated by comparing the labeling of $5 \mathrm{HT}$-immunoreactive neurons in third instar larval CNS (Fig. $1 B$ ) with first instar larval CNS (Fig. 3A).

We have previously described 5HT-immunoreactive fibers in two separate nerves (White and Vallés, 1985). Fibers filled with immunoreactive varicosities were found to project into the ring gland and the maxillary nerve.

\section{Development of larvae carrying genetic deletion of the Ddc gene}

The enzyme DDC in Drosophila is found in the CNS, where it is necessary for the synthesis of the neurotransmitters 5HT and dopamine (Dewhurst et al., 1972; Livingstone and Tempel, 1983; Wright, 1977). This enzyme activity is also found in the hypodermal cells, where DDC is an essential enzyme in the pathway leading to cuticle sclerotinization, in which dopamine is an important intermediate (e.g., see Wright, 1977). The DDC structural gene, $D d c$, of $D$. melanogaster, has been characterized genetically and molecularly (Hirsh and Davidson, 1981; Scholnick et al., 1983; Wright et al., 1976a, b). A number of $D d c$ genetic variants, point mutants, temperature-sensitive mutants, and deletions of the genetic locus are extant (Wright et al., 1976a, b, 1981). The DDC enzyme encoded by the $D d c$ gene has been shown to catalyze the decarboxylation of dihydroxyphenylalanine (DOPA) to dopamine and of 5-hydroxytryptophan (5HTP) to $5 \mathrm{HT}$ in the brain tissue (Livingstone and Tempel, 1983).

Animals deficient for the $D d c$ gene were generated using two deficiencies of the $D d c$ gene, $D f(2) 130$ and $D d c^{n 27}$; the genetic cross is described in Materials and Methods. A quarter of the progeny of this cross will be $D f(2) 130 / D d c^{n 27}$; these are referred to as either $D f D d c$ animals or as mutant animals in this paper. The phenotype of $D f D d c$ embryos is similar to the phenotype described by Wright et al. (1976b) for the mutant embryos of genotype $D d c^{n 7} / D f(2) 130 . D d c^{n 7}$ is an embryonic lethal allele at the $D d c$ locus (Wright et al., 1976b).

Most $D d c$ mutant animals die at the embryo-larval boundary and do not hatch (Wright et al., 1976b); however, a small number, approximately $1 / 100$, can hatch (T. R. F. Wright, personal communication). As larvae, the mutant escapers have incompletely sclerotized mouth hooks and setae, show varying degrees of postembryonic development, but do not eclose as adults ( $T$. R. F. Wright, personal communication). The $D f D d c$ pharate larvae, at late embryonic stages, appear similar to normal larvae. Moreover, the unhatched mutant larvae move vigorously within the embryonic membranes. We suspected that the majority of mutant embryos die at the embryo-larval boundary because of the failure of the pharate larvae to break out of the vitellin membrane. The failure to hatch could perhaps be due to the incompletely sclerotized mouth hooks of the mutant larvae, as originally suggested by Wright et al. (1976b). To obtain mutant animals in sufficient numbers and rear them under defined conditions, the mutant larvae were mechanically liberated.

To monitor the development of mechanically hatched mutant animals, each day the larvae were inspected and the larval instars were determined. As controls, heterozygous animals that hatched (naturally) at about the same time from the same cross, carrying one wild-type $D d c$ gene, were reared under similar conditions (control genotype: $D f(D d c) / C y O$; the control animals are referrred to as either wild-type or as control animals in this paper). Many of the $D f D d c$ larvae develop and undergo larval moults. However, the development of $D f D d c$ larvae was slower 


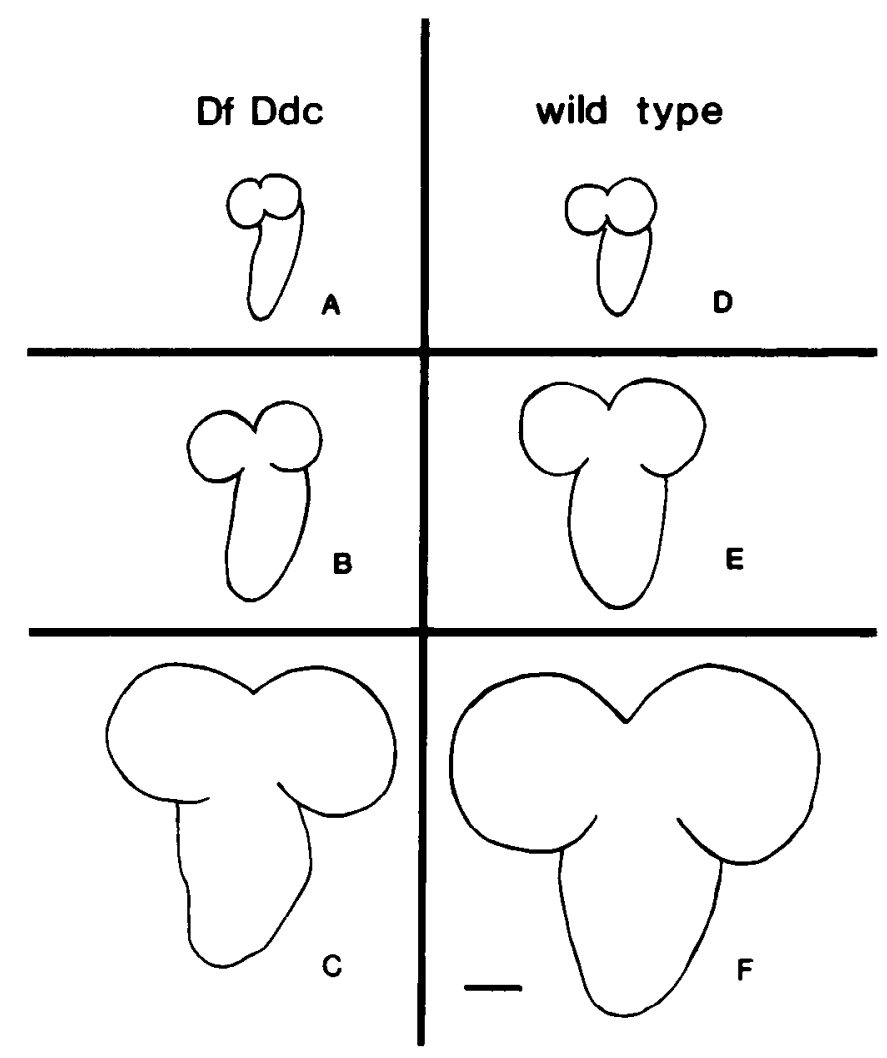

Figure 2. Growth of the $D f D d c$ and wild-type larval CNS. Schematic tracings adapted from photographs of larval CNSs depicting the overall size increase during larval development. CNSs of $D f D d c$ larvae. $A$, Newly hatched stage $(0 \mathrm{hr}) ; B$, late second instar stage $(72 \mathrm{hr}) ; C$, late third instar stage $(120 \mathrm{hr})$. CNSs of control larvae. $D$, Newly hatched stage $(0 \mathrm{hr}) ; E$, late second instar stage $(48 \mathrm{hr}) ; F$, late third instar stage $(96 \mathrm{hr})$. Axis same as in Figure 1. Scale bar, $50 \mu \mathrm{m}$.

when compared to control larvae reared similarly. A majority $(>90 \%)$ of the control larvae were third instar on the third day after hatching ( $72 \mathrm{hr}$ ), whereas the mutant larvae usually took at least $4 \mathrm{~d}(96 \mathrm{hr})$. The survival index, measured as the percentage of hatched larvae reaching third instar stage, was about $37 \%$ (41 third instar larvae/ 110 hatched larvac) for the mutant animals. The survival index for the control animals was $89 \%$ (49 third instar larvae/55 hatched larvae). Some of the observed $D f D d c$ mortality could be caused by injury suffered during teasing of the vitellin membrane. The hypoderm of these larvae is quite fragile, and an injury to the superficially located tracheal system can be fatal.

To determine if the CNSs of the $D f D d c$ animals grow during the larval period, CNSs from mutant and control animals from comparable developmental stages were dissected and photographed. The line drawings in Figure 2 show the overall size of the CNSs of the newly hatched, second, and third instar larvae from both mutants and controls. Although quantitative measurements on a large number of samples were not made, and some variation within each group in the size of the CNSs from chronologically similar animals was observed, two points can be made: (1) at hatching, the CNSs from mutant and control larvae were similar in overall dimensions (Fig. 2, $A, D$ ); (2) mutant CNSs grew as development progressed, as can be observed by the increase in dimensions (Fig. $2 A-C$ ).

\section{Absence of $5 H T$ in Df Ddc larval CNS}

To ascertain if the DDC enzyme encoded by the gene $D d c$ was the decarboxylase utilized in the 5HT-containing neurons, CNSs from third instar $D f D d c$ larvae were dissected and processed for 5HT immunocytochemistry (six samples). In all immunocytochemical studies described in this and subsequent sections, a monoclonal antibody against $5 \mathrm{HT}$ was used unless noted otherwise. CNSs from control larvae were processed in parallel (four samples). All the CNSs from the $D f D d c$ larvae were characterized by a total absence of immunoreactive staining of the neuronal somata and the neuropil (Fig. 3C). In addition to the lack of staining in the CNS, the peripheral immunoreactivity was also absent (Fig. 3, $B, C$ ). The immunoreactive staining of the control CNSs was normal.

To investigate if the CNSs were devoid of 5HT at earlier stages in development, CNSs were dissected and fixed immediately after hatching. In the wild-type (seven samples), the $5 \mathrm{HT}$-containing neurons are already present and immunoreactive at hatching (Fig. $3 A$ ). In contrast to the wild-type CNSs, those of the newly hatched $D f D d c$ larvae (six samples) were deficient in 5HT immunoreactivity (Fig. 3B). The lack of immunoreactive staining suggests a total absence of endogenous $5 \mathrm{HT}$ in the $D f D d c$ CNS.

\section{Neurons committed to $5 H T$ differentiation pathway are present in Df Ddc larval CNS}

Next, we addressed the question: Are neurons committed to the $5 \mathrm{HT}$ differentiation pathway present in the $D f D d c$ larval CNS? By commitment to the $5 \mathrm{HT}$ pathway we imply that these neurons will contain $5 \mathrm{HT}$ and express at least one other biochemical property characteristic of 5HT-containing neurons. The property we chose to look at was the selective uptake capability of the monoamine-containing neurons (Axelrod, 1965; Ross, 1982). Serotonin-containing neurons have been shown to take up 5HT selectively (e.g., Glover, 1984; Osborne et al., 1975).

$D f D d c$ larval CNSs were incubated with $10^{-6} \mathrm{M} 5 \mathrm{HT}$ as described in Materials and Methods. To visualize sequestered 5HT, the CNSs were processed for 5HT immunocytochemistry. As controls, mutant CNSs incubated in Ringer's alone and control CNSs incubated in the presence and absence of exogenous 5HT were treated similarly. The results are summarized below.

1. In the control CNSs (seven samples), the 5HT-immunoreactive neuronal pattern remained unaltered when incubated with exogenous $5 \mathrm{HT}$. Figure $4 A$ shows a representative wholemount. This result indicates that in the wild-type CNS, under the experimental conditions used, non-5HT-containing neurons do not exhibit immunocytochemically detectable 5HT uptake (Fig. 4A).

2. Serotonin-immunoreactive cells were observed in the $D f$ $D d c$ CNSs (12/13 samples) incubated in exogenous 5HT, indicating that at least some neurons in the mutant CNS are capable of accumulating 5HT (referred to as selective 5HT uptake neurons) and that the 5HT uptake is selective (Fig. $4 B$ ). Control mutant CNSs (four samples) incubated in Ringer's alone were devoid of immunoreactivity (data not shown).

3. The pattern of neurons in the individual mutant CNSs revealed through the uptake of 5HT is represented in Table 2. The pattern was somewhat variable and will be described in more detail below. However, in the segments in which selective 5HT uptake neurons were evident, their numbers, somata position, and pattern of primary projections were highly reminiscent of the normal 5HT-immunoreactive neuronal pattern (Fig. $4, A, B)$.

4. In the third instar mutant larval CNSs, selective 5HT uptake neurons were consistently discerned in the subesophageal (12/13 samples) and thoracic segments ( $12 / 13$ samples). In most samples, neurons were observed in the abdominal segments (11/ 13 samples). In general, in the ventral ganglion, neurons in the anterior segments were more brightly stained than the neurons in the posterior segments. Also, selective 5HT uptake neurons were usually not observed in the brain lobes, and when they were, the staining was very weak ( $1 / 13$ samples). 
5. In samples where the maxillary nerve-pharyngeal muscle complex was left intact with the mutant CNS, immunoreactivity in the nerve, the plexus, and pharangeal muscle was observed ( $9 / 12$ samples).

The similarities between the patterns of selective 5HT uptake neurons and the normal 5HT-immunoreactive neurons suggest that in the $D f D d c$ larval CNS: (1) the labeled neurons appear to be the same as those that are normally committed to the 5HT differentiation pathway in the wild-type animals and (2) at least some (and probably all) neurons able to take up 5HT persist in the larval CNS.

\section{All embryonic neurons initially committed to the $5 H T$ differentiation pathway show selective 5HT uptake}

As described above, not all 5HT-immunoreactive neurons were revealed after incubating the $D f D d c$ third instar larval CNS in exogenous 5HT. In general, the neurons in the ventral ganglion were able to accumulate $5 \mathrm{HT}$ at an immunocytochemically detectable level, while those in the brain lobes were not (Table 2). To test if the differential in the ability to take up 5HT observed in the third instar CNSs was also characteristic of the $D f D d c$ CNSs at earlier developmental stages, the uptake experiments were performed with the $D f D d c$ larval CNSs dissected from larvae that were (1) pharate larvae, (2) young second instar larvae, and (3) larvae close to the second/third instar moult boundary (i.e., late second instar or young third instar).

To determine the selective 5HT uptake neuronal pattern in the newly hatched $D f D d c$ larvae, CNSs were dissected from larvae that were still in the embryonic membranes (five samples). In these young CNSs, selective 5HT uptake was evident in all the neurons committed to the 5HT differentiation pathway (Fig. 4C, Table 2). In contrast to what was observed with the older CNSs, at this stage the neurons in the brain lobes were capable of sequestering 5HT. In four out of five samples, the entire neuronal complement was revealed after incubation in 5HT. Only in one sample was the pattern incomplete, possibly because of damage during handling, although even in this sample, the neurons in the brain lobes were present (Table 2). The pattern of selective 5HT uptake neurons in the early second instar CNSs (four samples) was similar to the pattern in the newly hatched larval CNS, except that in one sample some variation in the staining of abdominal neurons was observed (Table 2).

In CNSs dissected from the second/third instar larvae, 5HT uptake neurons were found in all samples (13 samples). In 8 of these, 5HT uptake neurons were detected in all the neuromeres of the ventral ganglion and the pattern was identical to the wildtype 5HT-immunoreactive neuronal pattern, except that uptake by brain lobe neurons was not observed. Figure $4 D$ is an example of the 5HT uptake neuronal pattern observed in the second/ third instar larval CNSs ( $8 / 13$ samples). The $5 \mathrm{HT}$ uptake neurons revealed in individual CNSs at different developmental stages are presented in Table 2.

In summary, the studies of 5HT uptake neurons at different developmental stages suggest that in the $D f D d c$ CNSs, all embryonic neurons committed to the $5 \mathrm{HT}$ pathway initially show normal differentiation of the selective uptake properties. However, as development continues an apparent loss in the ability to take up 5HT is observed in some parts of the CNS.

\section{Older CNSs are less permeable to exogenous $5 H T$}

The 5HT uptake studies with the older mutant CNSs (second/ third instar and third instar) show a clear distinction in the 5HT uptake properties of the brain lobe and the ventral ganglion (Table 2). One factor that can contribute to the observed loss in the ability to take up 5HT by some of the neurons is a possible progressive lack of access of the externally applied amine into the CNS.
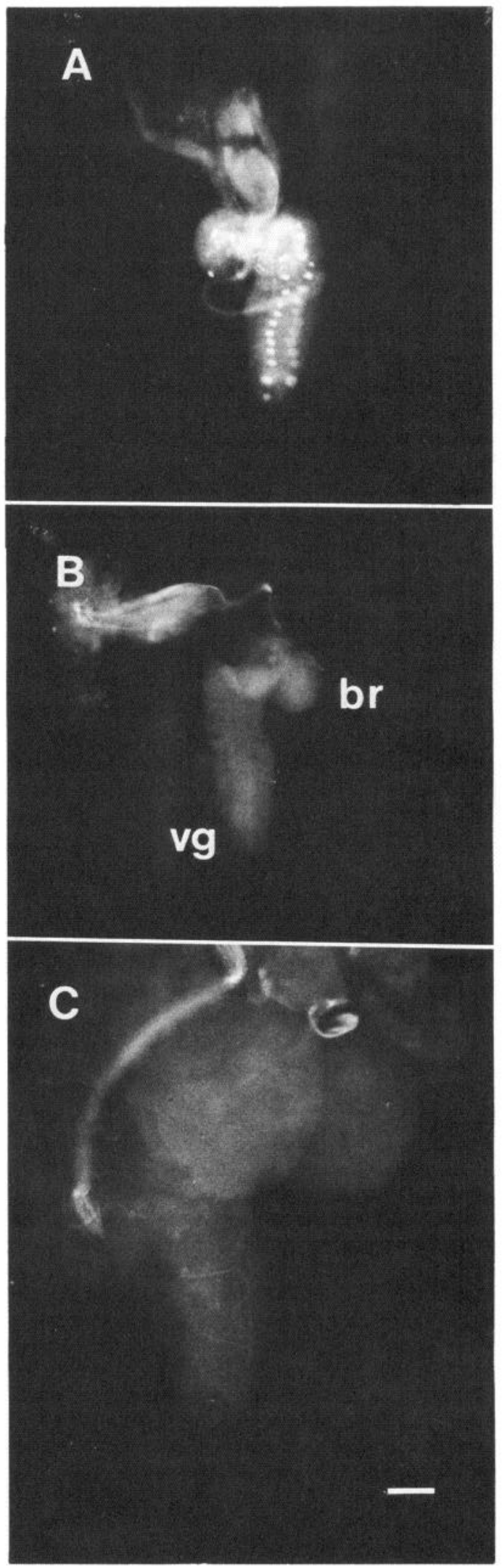

Figure 3. Serotonin immunoreactivity in wild-type and $D f D d c$ CNS. Photomicrographs of immunofluorescently stained larval CNS. $A$, Wildtype CNS of a newly hatched larva; $B, D f D d c$ CNS of a newly hatched larva; $C, D f D d c$ CNS of a third instar larva. Note the complete absence of 5HT immunoreactivity in the brain and ventral ganglion of both the newly hatched and third instar larval CNS samples. $b r$, brain, $v g$, ventral ganglion. Axis as in Figure 1. Scale bar, $50 \mu \mathrm{m}$. 


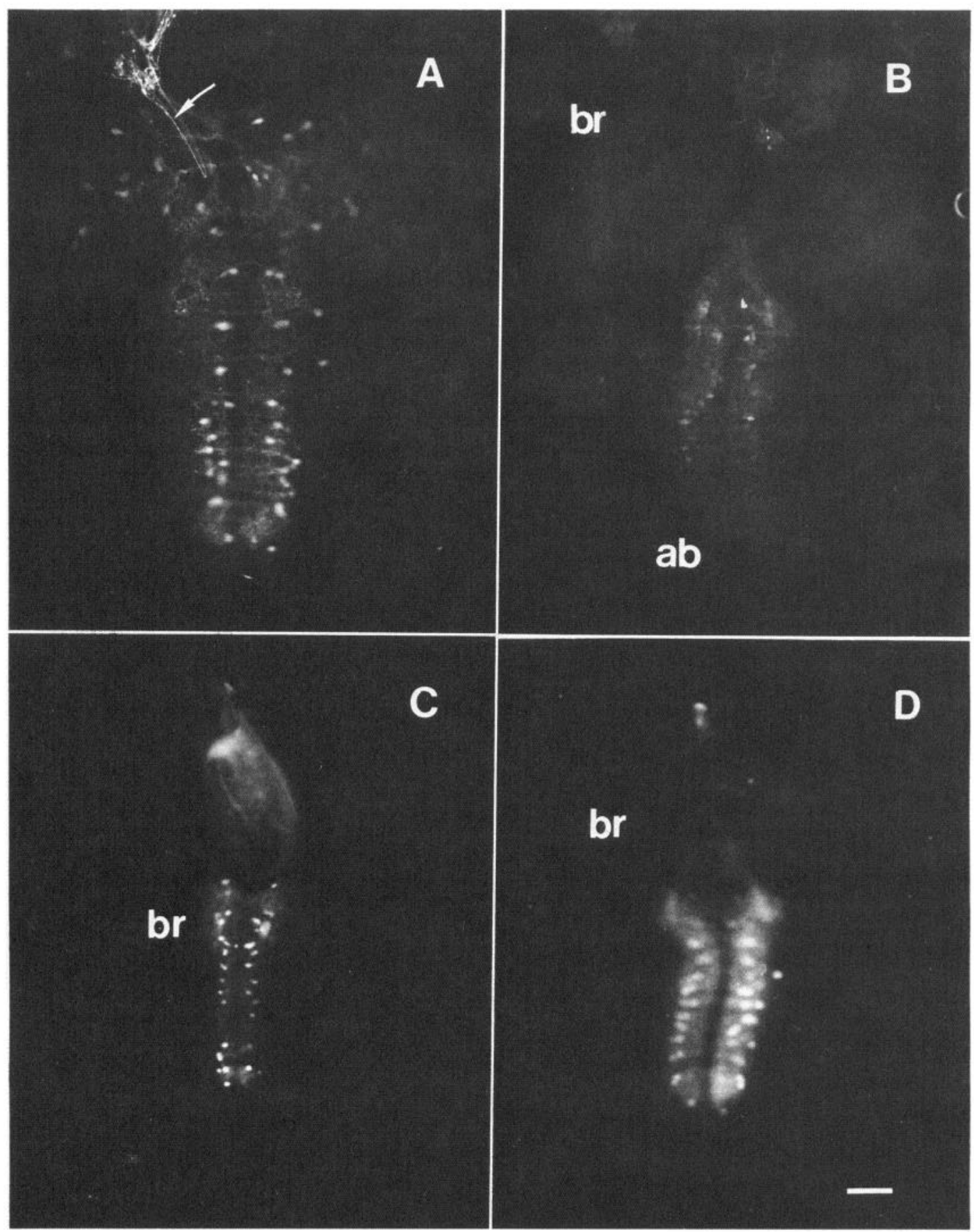

Figure 4. Serotonin immunoreactivity in wild-type and mutant CNS after incubation in exogenous 5HT. Photomicrographs of larval CNS after immunofluorescent staining. $A$, Control wild-type CNS from third instar larva. The arrow points to immunoreactive fibers leaving from the dorsal midbrain to the ring gland. $B, D f D d c$ third instar CNS. Note the absence of the 5HT uptake neurons in the brain and the posterior abdominal segments. $C$, Early second instar $D f D d c$ CNS. Note the presence of 5 HT uptake neurons in the brain. $D$, Late second/early third instar $D f D d c$ CNS. Note the absence of 5 HT uptake neurons in the brain. This sample was immunoreacted with a polyclonal antibody. $b r$, Brain, $v g$, ventral ganglion. Axis as in Figure 1. Scale bar, $50 \mu \mathrm{m}$.

To test this possibility, the CNSs dissected from mutant third instar larvae were broken to allow better penetration and then incubated in 5HT. Typically, a cut was made between the paired brain lobes, freeing one lobe from the rest of the CNS. The free brain lobes were processed along with control intact $D f D d c$ CNSs. At least some selective 5HT uptake neurons were observed in each of the free brain lobe samples (four samples; see Table 3). The control CNSs showed a selective 5HT uptake pattern typical of the intact third instar CNS (compare control samples from Table 3 to third instar samples from Table 2). Figure $5 A$ is a micrograph of a broken brain lobe after uptake, and Figure $5 B$ is a micrograph of a brain lobe from a control intact CNS sample processed with it. Comparison of these 5HT immunoreactivities clearly indicates that the brain lobe neurons in the intact samples do not take up 5HT, while the brain lobe neurons in the broken sample do. Presumably this difference is due to the inability of the exogenous $5 \mathrm{HT}$ to readily penetrate through the older CNS. 
Table 2. Serotonin uptake neuronal clusters evidenced in $D f D d c$ larval CNSs

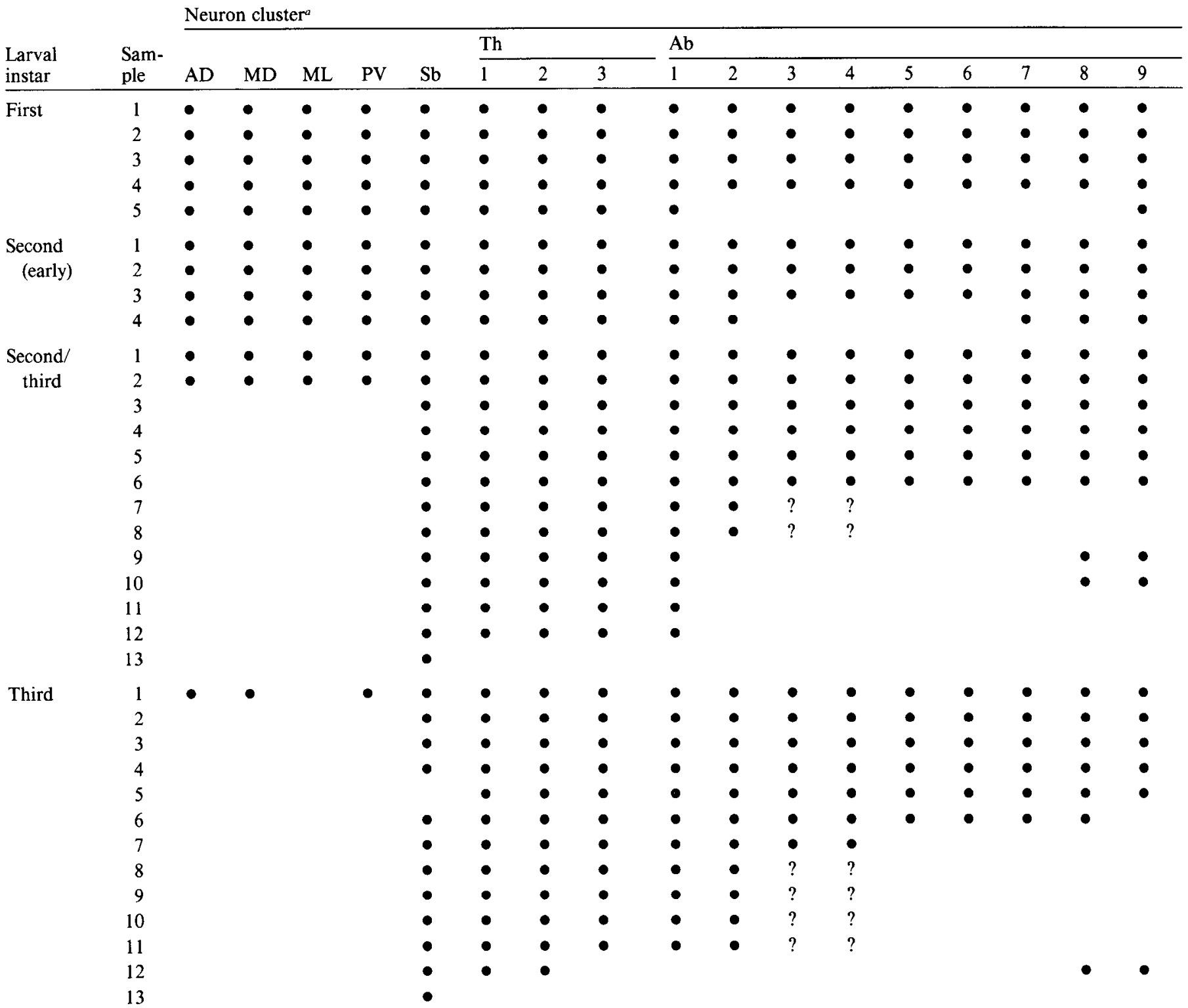

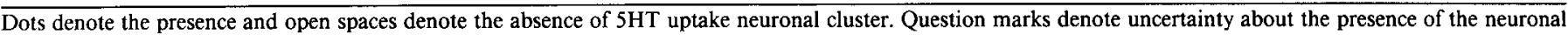
cluster.

${ }^{a}$ For localization of neuronal clusters, see Table 1.

These observations of selective 5HT uptake on broken nervous tissue imply that the differences in the uptake properties of the neurons in the older CNSs evidenced in the intact samples (Tables 2,3 ) are due to unavailability of exogenous 5HT to the neurons in the brain lobes.

\section{Discussion}

\section{Immunocytochemical method}

In this study we depended entirely on immunocytochemical detection of in situ 5HT; thus, all the results are subject to the limitations of the immunocytochemical methodology (e.g., discussed in Bishop and O'Shea, 1982; Hökfelt et al., 1980). The two important points to consider are the specificity and the sensitivity of the reaction. With regard to the specificity, the monoclonal antibody used in this study (1) specifically reveals neurons known to contain 5HT in rat brain sections (Conso- lazione et al., 1981); (2) yields the same immunoreactivity pattern in the Drosophila larval CNS as 5HT antiserum from Immunonuclear Corporation (White and Vallés, 1985); and (3) fails to show any immunoreactivity in $D f D d c$ CNSs. Therefore, we are fairly confident that the antibody is specific for 5HT. However, we do not know the sensitivity of the immunocytochemical assay.

Gene Ddc encodes the DDC utilized in the

$5 H T$-immunoreactive neurons

Livingstone and Tempel (1983) have suggested that the gene $D d c$ encodes a DDC activity in the brain utilized in the conversion of 5HTP to 5HT. To study the effect of $D d c$ mutations on the synthesis of 5HT and dopamine, they utilized a temperature-sensitive allele $D d c^{t s}$ (Livingstone and Tempel, 1983). In agreement with Livingstone and Tempel (1983), we had previously observed a decrease in the intensity of 5HT immuno- 


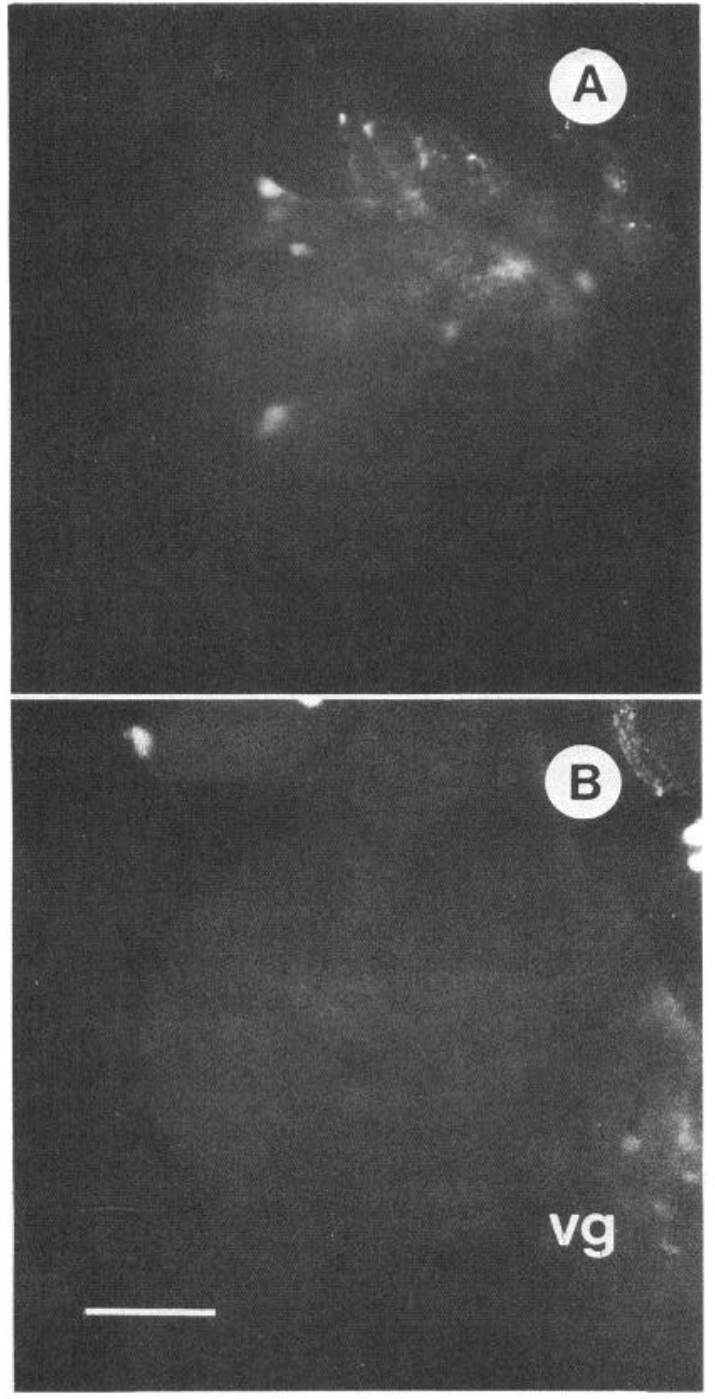

Figure 5. Serotonin-immunoreactive neurons in $D f D d c$ brain lobes from broken and intact CNSs after incubation in exogenous 5HT. A, Broken brain lobe from a $D f D d c$ larval CNS. Note the presence of selective $5 \mathrm{HT}$ uptake neurons in the brain lobe and immunoreactivity in the neuropil. $B$, Brain lobe from an intact $D f D d c$ larval CNS. Note the absence of immunoreactivity from the brain lobe. $v g$, Ventral ganglion. Scale bar, $50 \mu \mathrm{m}$.

reactivity in the CNSs of $D d c$ temperature-sensitive mutants, although we observed some residual immunoreactivity as well (White and Vallés, 1985). The presence of a second decarboxylase in 5HT-containing neurons, perhaps with a lower affinity for 5HTP, could have explained the incomplete block of immunoreactive staining in the temperature-sensitive mutants (alternative explanations discussed in White and Vallés, 1985). The complete block of 5HT-immunoreactive staining observed in the $D f D d c$ CNS strongly suggests that the gene $D d c$ encoded DDC is the only decarboxylase utilized in the 5HT-containing neurons.

The absence of detectable 5HT immunoreactivity in $D f D d c$ CNS is presumably the direct result of the absence of the DDC enzyme in the differentiated 5HT-containing neurons. In such neurons, the absence of DDC could cause an accumulation of $5 \mathrm{HTP}$, the immediate precursor to $5 \mathrm{HT}$. We used the monoclonal antibody because, according to the specificity studies reported by Consolazione et al. (1981), preabsorption of the antibody with 5 HTP had no effect on specific immunofluorescence
Table 3. Serotonin uptake neuronal clusters from isolated brain lobes of $D f D d c$ larval CNSs

\begin{tabular}{llllllll}
$\begin{array}{l}\text { Sam- } \\
\text { ple }\end{array}$ & \multicolumn{2}{l}{ Neuron cluster } \\
\cline { 2 - 7 } & $\mathrm{AD}$ & $\mathrm{MD}$ & $\mathrm{ML}$ & $\mathrm{PV}$ & $\mathrm{Sb}$ & $\mathrm{Th} 1$ & $\mathrm{Th} 2$ \\
\hline $\begin{array}{l}\text { Broken lobe } \\
1\end{array}$ & $\bullet$ & $\bullet$ & $\bullet$ & $\mathrm{ag}$ & $\mathrm{ag}$ & $\mathrm{ag}$ \\
2 & & $\bullet$ & $\bullet$ & $\bullet$ & $\mathrm{ag}$ & $\mathrm{ag}$ & $\mathrm{ag}$ \\
3 & $\bullet$ & $\bullet$ & $?$ & $?$ & $\mathrm{ag}$ & $\mathrm{ag}$ & $\mathrm{ag}$ \\
4 & $\mathrm{~b}$ & $\mathrm{~b}$ & $\mathrm{~b}$ & $\mathrm{~b}$ & $\mathrm{ag}$ & $\mathrm{ag}$ & $\mathrm{ag}$ \\
Control & & & & & & \\
1 & & $\bullet$ & & $\bullet$ & $\bullet$ & $\bullet$ \\
2 & & & & $\bullet$ & $\bullet$ & $\bullet$ \\
3 & & & & $\bullet$ & $\bullet$ & $\bullet$ \\
4 & & & & $\bullet$ & $\bullet$ & $\bullet$
\end{tabular}

Dots denote the presence and open spaces the absence of 5HT uptake neuronal cluster. Question marks denote uncertainty about the presence of the neuronal cluster. ag indicates the ganglion was absent. b indicates that 5HT uptake neurons were observed, but they could not be assigned to specific clusters.

${ }^{a}$ For localization of neuronal clusters, see Table 1.

and 5HTP was a poor inhibitor in an agglutination assay. Because of the lack of cross-reactivity with 5HTP, the monoclonal antibody is not likely to detect neurons that may have accumulated 5HTP. It is pertinent to mention in this context that the polyclonal 5HT antiserum we previously used (White and Vallés, 1985) appears to cross-react weakly with 5HTP because in $D f D d c$ CNSs faintly stained neurons were observed (A. M. Vallés, unpublished observations). Preabsorption of the antiserum with 5HTP blocked the weak staining of the mutant CNS but had no effect on the staining of the wild-type (A. M. Vallés, unpublished observations).

\section{Postembryonic development of Df Ddc larvae}

Most animals deficient in the enzyme DDC fail to hatch (Wright et al., 1976b). Wright et al. (1976b) have suggested that the inability of the pharate larva to crawl out of the embryonic membrane could be due to the incompletely sclerotized epidermis. Our observation that $30-40 \%$ of mechanically hatched Df $D d c$ larvae show continued development, molt, and even attempt to pupate is consistent with the idea that it is the epidermal DDC deficiency that is responsible for the embryonic lethality.

It is indeed surprising that animals deprived of both 5HT and dopamine (Livingstone and Tempel, 1983; Wright, 1977), two major monoamine transmitters, can develop as far as the late larval stages (6-7 d after hatching). This is in contrast to what is observed with animals deficient in the enzyme choline acetyltransferase, which is essential for the synthesis of the neurotransmitter ACh. Greenspan (1980) observed that zygotes carrying mutations in the structural gene for the enzyme choline acetyltransferase, Cha, die at the embryo-larval boundary, and if an occasional larva manages to crawl out of the embryonic membranes, it dies immediately.

The survival of $D f D d c$ larvae implies that the neurotransmitters 5HT and dopamine are not critically involved in functions essential to the viability of the organism, at least until pupation. Since DDC activity is found in small amounts in ovarian tissues (Wright et al., 1981), it is possible that the zygote has maternally stored pools of these neurotransmitters and/or has maternally stored DDC enzyme and/or has maternally stored $D d c$ messenger RNA. In the case of $5 \mathrm{HT}$, the maternal pool may be below the detection level of immunocytochemical meth- 
od. These maternally contributed products (if any) may be sufficient during embryonic and larval period.

\section{$5 H T$-containing neurons differentiate in absence of endogenous $5 H T$}

The 5HT uptake studies performed with the newly hatched mutant CNSs show that the 5HT-containing neurons differentiate in normal numbers and positions during embryogenesis in the absence of immunocytochemically detectable 5HT. This normal pattern is not surprising in light of what is known of the developmental history of 5HT-containing neurons in other invertebrates. Taghert and Goodman (1984) have shown that in the grasshopper embryonic CNS, axonogenesis and pathway choices precede immunocytochemical staining of 5HT-containing neurons, and the morphogenesis of the neurons continues after transmitter expression. In Drosophila, the 5HT-containing neurons can be reliably immunocytochemically stained in 18 hr embryonic CNS (A. M. Vallés, unpublished observations) several hours after the appearance of DDC enzyme activity in whole embryos (Beall and Hirsh, 1984; Gietz and Hodgetts, 1985). Our studies show that the primary projection pattern in the mutant CNS is similar to that of the wild-type. The methods we used in this study did not have the resolution necessary to determine if the final dendritic arborizations of individual neurons are identical in the wild-type and the mutant CNSs.

The neurons committed to 5HT differentiation pathway can be revealed through their selective uptake properties in the mutant CNSs. This observation implies that these neurons continue to express the genes necessary for the selective uptake mechanism in the absence of immunocytochemically detectable endogenous $5 \mathrm{HT}$.

\section{Access of exogenous $5 H T$ into the mutant CNS appears to be progressively blocked}

When intact young mutant CNSs (newly hatched) are incubated with the amine, selective 5HT uptake neurons in all parts of the CNS are revealed, but in similarly treated older mutant CNSs, uptake of 5HT in neurons located in the brain lobes is not observed (Table 2, Fig. 4). However, if the CNS is broken to facilitate the entry of the amine, the neurons located in the brain lobe exhibit selective 5HT uptake (Table 3, Fig. 5). It appears likely that at least in the case of the mutant, in younger CNSs, 5HT can penetrate through the sheath that envelops the CNS, but that it gets progressively harder for the $5 \mathrm{HT}$ to penetrate the sheath of older CNSs.

In the older intact CNSs, 5HT is available at least to some cells (Table 3, Fig. $4 B, D$ ). A possible explanation is that $5 \mathrm{HT}$ is transported by the 5HT-containing peripheral nerves back to the CNS. Indeed, when we incubated dissections in which the maxillary nerve and the pharyngeal muscle were left connected to the CNS, or the dissections had the ring gland still attached to the CNS, immunoreactive fibers and plexus were observed in the periphery. The ability of the peripheral nerves to take up 5HT might allow one to argue that the $5 \mathrm{HT}$ available to the subesophageal neurons entered the CNS through the maxillary nerve. One inconsistency in this explanation is that, by similar argument, some neurons in the brain lobes should have access to $5 \mathrm{HT}$ through the ring gland fibers, yet none of the brain lobe neurons showed accumulation of $5 \mathrm{HT}$.

Finally, we would like to point out that it is indeed possible that other biochemical and/or morphological changes occur in the CNSs of mutant larvae. In this report we have only focused on 5HT-containing neurons as assayed by $5 \mathrm{HT}$ immunocytochemistry. It will be interesting to determine whether in the $D f$ $D d c$ CNS, changes occur in catecholamine-containing neurons, neurons that are target neurons, for the serotonergic and catecholaminergic neurons, and neurons that might be able to provide compensatory functions.

\section{References}

Axelrod, J. (1965) The metabolism, storage and release of catecholamine. Recent Prog. Horm. Res. 21: 597-622.

Beall, C. J., and J. Hirsh (1984) High levels of intron-containing RNAs are associated with expression of the Drosophila dopa decarboxylase gene. Mol. Cell. Biol. 4: 1669-1674.

Bishop, C. A., and M. O'Shea (1982) Neuropeptide proctolin (H-ArgTyr-Leu-Pro-Thr-OH): Immunocytochemical mapping of neurons in the central nervous system of the cockroach. J. Comp. Neurol. 207: 223-238.

Bodenstein, D. (1950) The postembryonic development of Drosophila. In Biology of Drosophila, M. Demerec, ed., pp. 275-367, Hafner, New York.

Budnik, V., A. M. Vallés, and K. White (1985) Histofluorescence of catecholamine-containing neurons in Drosophila. Soc. Neurosci. Abstr. 11: 944.

Consolazione, A., C. Milstein, B.Wright, and A. C. Cuello (1981) Immunocytochemical detection of serotonin with monoclonal antibodies. J. Histochem. Cytochem. 29: 1425-1430.

Dewhurst, S. A., S. G. Croker, K. Ikeda, and R. E. McCaman (1972) Metabolism of biogenic amines in Drosophila nervous tissue. Comp. Biochem. Physiol. 43B: 975-981.

Gietz, R. D., and R. B. Hodgetts (1985) An analysis of dopa decarboxylase expression during embryogenesis in Drosophila melanogaster. Dev. Biol. 107: 142-155.

Glover, J. C. (1984) Structure, function and development of serotonincontaining neurons in the leech. Ph.D. thesis, University of California, Berkeley.

Greenspan, R. J. (1980) Mutations of choline acetyltransferase and associated neural defects in Drosophila melanogaster. J. Comp. Physiol. 137: 83-92.

Harris, W. A. (1981) Neural activity and development. Annu. Rev. Physiol. 43: 689-710.

Haydon, P. G., D. P. McCobb, and S. B. Kater (1984) Serotonin selectively inhibits growth cone motility and synaptogenesis of specific identified neurons. Science 226: 561-564.

Hirsh, J., and N. Davidson (1981) Isolation and characterizaton of the dopa decarboxylase gene of Drosophila melanogaster. Mol. Cell. Biol. 1: 475-485.

Hökfelt, T., O. Johansson, A. Ljungdahl, J. M. Lundberg, and M. Schultzberg (1980) Peptidergic neurones. Nature 284: 515-521.

Lindsley, D. L., and E. H. Grell (1968) Genetic Variations of Drosophila melanogaster. Carnegie Institution of Washington Publ. 627, Baltimore.

Livingstone, M. S., and B. L. Tempel (1983) Genetic dissection of monoamine neurotransmitter synthesis in Drosophila. Nature 303: $67-70$.

Osborne, N. N., L. Hiripi, and V. Neuhoff (1975) The in vitro uptake of biogenic amines by snail (Helix pomatia) nervous tissue. Biochem. Pharmacol. 24: 2141-2148.

Ross, S. B. (1982) The characteristics of serotonin uptake systems. In Biology of Serotonergic Transmission, N. N. Osborne, ed., pp. 159196, Wiley, New York.

Scholnick, S. B., B. A. Morgan, and J. Hirsh (1983) The cloned dopa decarboxylase gene is developmentally regulated when reintegrated into the Drosophila genome. Cell 34: 37-45.

Taghert, P. H., and C. S. Goodman (1984) Cell determination and differentiation of identified serotonin-immunoreactive neurons in the grasshopper embryo. J. Neurosci. 4: 989-1000.

Vallés, A. M., and K. White (1985) Serotonergic cells in Drosophila. Soc. Neurosci. Abstr. 11:958.

White, K., and A. M. Vallés (1985) Immunohistochemical and genetic studies of serotonin and neuropeptides in Drosophila. In Molecular Bases of Neural Development, G. M. Edelman, W. E. Gall, and W. M. Cowan, eds., pp. 547-563, Wiley, New York.

White, K., T. Hurteau, and P. Punsel (in press) Neuropeptide FMRFlike immunoreactivity in Drosophila: Development and distribution. J. Comp. Neurol.

Wright, T. R. F. (1977) The genetics of dopa decarboxylase and $\alpha$-methyl dopa sensitivity in Drosophila melanogaster. Am. Zool. 17: 707-721.

Wright, T. R. F., R. B. Hodgetts, and A. F. Sherald (1976a) The genetics of dopa decarboxylase in Drosophila melanogaster. I. Isolation and characterization of deficiencies that delete the dopa-de- 
carboxylase-dosage-sensitive region and the $\alpha$-methyl-dopa-hypersensitive locus. Genetics 84: 267-285.

Wright, T. R. F., G. C. Bewley, and A. F. Sherald (1976b) The genetics of dopa decarboxylase in Drosophila melanogaster. II. Isolation and characterization of dopa-decarboxylase-deficient mutants and their relationship to the $\alpha$-methyl-dopa-hypersensitive mutants. Genetics 84: 287-310.
Wright, T. R. F., R. Steward, K. W. Bentley, and P. A. Adler (1981) The genetics of dopa decarboxylase in Drosophila melanogaster. III. Effects of a temperature sensitive dopa decarboxylase deficient mutation on female fertility. Dev. Genet. 2: 223-235. 